\title{
Mura urbiche nell'Italia centro-settentrionale: significato e funzione tra la fine della repubblica e la prima età imperiale
}

\author{
Dario Canino \\ Sapienza Università di Roma \\ email: caninodario@gmail.com
}

\begin{abstract}
The text synthesizes an analysis of the meaning and function of city walls through a series of examples of urban settlements founded, or renewed, in cental-northern Italy, between the end of the Republic and the First Imperial Age. A period in which, in different geographical areas, different monumental results were produced. In some cases the need for defense is still relevant and the structure of city walls, gates and towers, reveal this need. Despite the Pax Augusta, the phenomenon of brigandage, in primis, imposes in some areas a certain attention to their defensive function. In other cases, the city walls may have existed to perform functions different than that of defensive; functions that are not limited only to a symbolic ritual matrix. In this process, even the city gates are transformed, entering in symbiosis with the monumental typology of the honorary arch and becoming, sometimes, a real commemorative monument of particular events. The prestige of the location of this monumental typology in the city wall is completely independent from the validity of the defensive walls. The city walls interact with the doors and with the towers both functionally and formally. Now, their task is not to close the village in a rigid internal/external alternative but rather to create a dialogue between the reality of the settlement and what surrounds it. This dialogue takes place, thanks to the doors and to other structures, both man-made, such as bridges, or natural, such as courses of water, paying attention to the element of the spatial perspective, that emerges, now, in many cases of urban renewal and thanks

to which is created an interaction between the city and the outside world.

Keywords: City Walls, City Gates, Town Planning, Perspective, Roman Cities

Testo

Ad eccezione di Roma, dove i resti delle mura ormai da tempo venivano abbattuti, in molte città romane si rilevano iniziative, sia tardorepubblicane sia augustee e giulio-claudie, volte alla realizzazione di restauri, ristrutturazioni ed anche nuove costruzioni di cinte urbane. In molti casi si tratta di opere la cui realizzazione, da una parte, era necessaria, a causa delle distruzioni che coinvolsero numerose città negli anni della guerra sociale (91-88 a.C.) e della guerra civile (49-45 a.C.), il cui ricordo era indubbiamente ancora troppo fresco per essere dimenticato; dall'altra parte, la loro realizzazione era anche voluta, per motivazioni di ordine religioso e giuridico che pesarono altrettanto sulle collettività, spingendole ad impegnare risorse considerevoli per il restauro o la costruzione delle mura urbiche.

Da sempre, e fino a questo momento, le mura urbiche avevano avuto un senso e un significato di cesura, di rapporto interrotto tra insediamento circoscritto e territorio adiacente; determinando l'originaria e fondamentale distinzione tra spazio interno e spazio esterno, separando la sfera dell'abitato, limitata e conosciuta, dalla dimensione della campagna, indefinita e diversa. Tale differenza, che distingue le due entità spaziali,
\end{abstract}




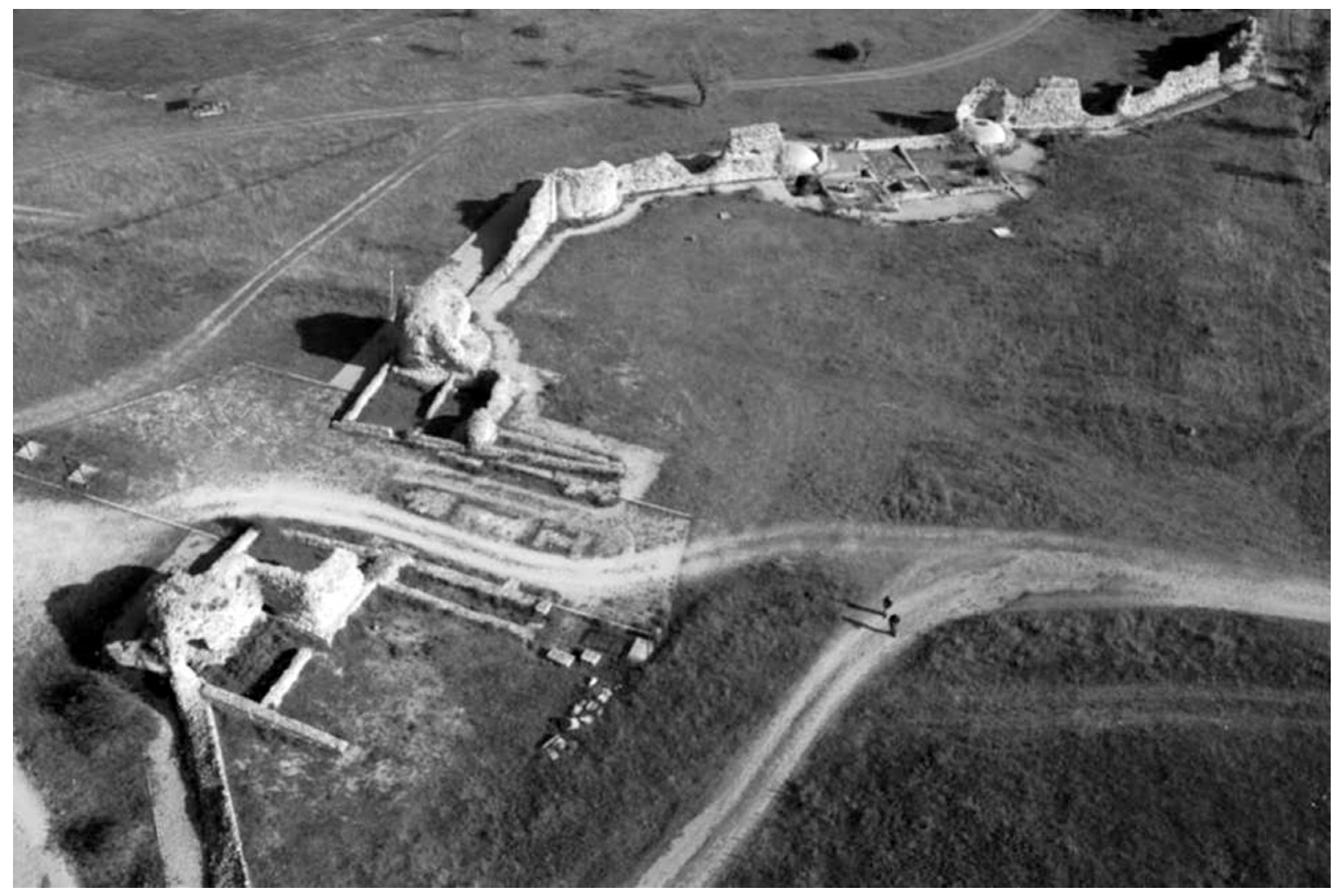

Fig. 1. Peltuinum. La porta occidentale. È ancora leggibile la struttura a doppio fornice con cavedio, protetta dalle due torri avanzate e la successiva chiusura dell'interturrio per mezzo dei muri della doganella (da Migliorati, 2014: 253).

da una parte e dall'altra delle mura, trovava, nel mondo romano, una conferma palese nella stessa destinazione in area extraurbana dei luoghi riservati alla sepoltura, ossia le necropoli, che erano le città dei morti, poste lungo la strada che consentiva il passaggio tra città dei vivi e agro (Rosada, 1990: 365).

La diffusione coloniale augustea, come anche la precedente triumvirale, mostra che, a fronte di poche città di impianto ex novo, la maggior parte dei centri coinvolti rientra in un quadro di ristrutturazione urbanistica o di recente programmazione e, dunque, ancora in fase di completamento edilizio, certamente codificato negli aspetti formali secondo la normativa urbana che è patrimonio degli anni centrali dell'ultimo secolo della Repub- blica. Le varie circostanze storico-politiche, che interessarono in tempi e modi specifici le diverse regioni, determinarono atteggiamenti differenti da parte del potere centrale nei confronti del problema della difesa del territorio e delle città.

Esistono casi di costruzione ex novo di nuovi perimetri murari; tra questi casi troviamo le nuove fondazioni augustee come, ad esempio, Augusta Taurinorum e Augusta Prætoria Salassorum. Queste città, insieme alle altre nuove fondazioni e alle città rifondate in questi anni, o che comunque raggiungono vera dignità civica solo in epoca augustea, ovviamente, forniscono la maggiore quantità d'informazioni sull'argomento, poiché rivelatrici del messaggio che il costruttore ha inteso imprimere nella struttura nel momento in cui questa è stata concepita o rinnovata.

Molte variabili hanno rivestito un ruolo di notevole importanza nelle scelte urbanistiche attuate, come, ad esempio: aemulatio municipalis, evergetismo, vicinanza all'Urbs, imitatio Urbis, ma anche controllo fiscale, viabilità e collegamenti con altri insediamenti, interazione con il paes- 


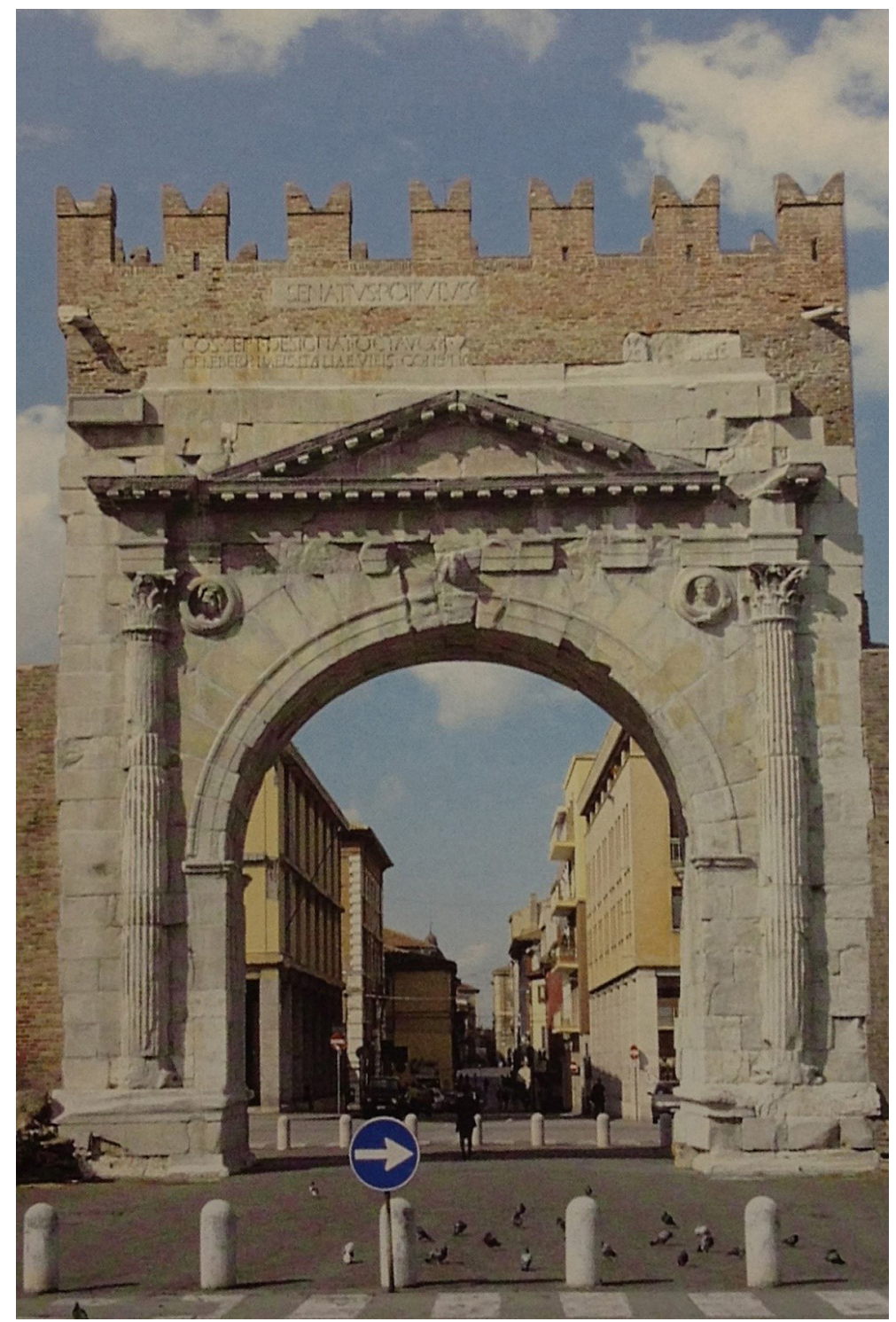

Fig. 2. Rimini (Ariminum). L'arco di Augusto come appare oggi (da Foschi \& Pasini, 1998: 36).

aggio circostante e, in particolare, con la realtà orografica e idrografica e, non in ultimo, ragioni di sicurezza legate, in primis, al mai cessato pericolo del brigantaggio.

La cinta, infatti, non ha solo una funzione di definizione strutturale; non meno importanti continuano ad essere le ragioni di sicurezza, considerato che, alla metà del I sec. a.C., molti comprensori dell'Italia pativano le conseguenze del brigantaggio e dei movimenti servili e la città murata, come testimonia Cicerone (Cluent., 195), è vista come un rifugio necessario per gli abitanti delle campagne circostanti. Un'attenzione particolarmente indirizzata alla funzione difen- siva viene rivelata da alcune caratteristiche che si presentano, ad esempio, nelle cinte urbane di Saepinum, il cui progetto deve risalire all'ultimo decennio del I sec. a.C. (Matteini Chiari, 1982), e Peltuinum (Fig. 1), di età cesariana. Le due città erano difese, infatti, da un alto numero di torri la cui dislocazione era stata progettata per rispondere a reali esigenze strategiche, e non a regole urbanistiche e di simmetria, impostandosi negli snodi tra i tratti murari, al fine di garantire una più efficace difesa, soprattutto dei punti più vulnerabili, come le porte (Casazza \& Pansini, in press; Migliorati, 2014; Migliorati \& Canino, 2014). Un aspetto molto rilevante, collegato alle cinte 


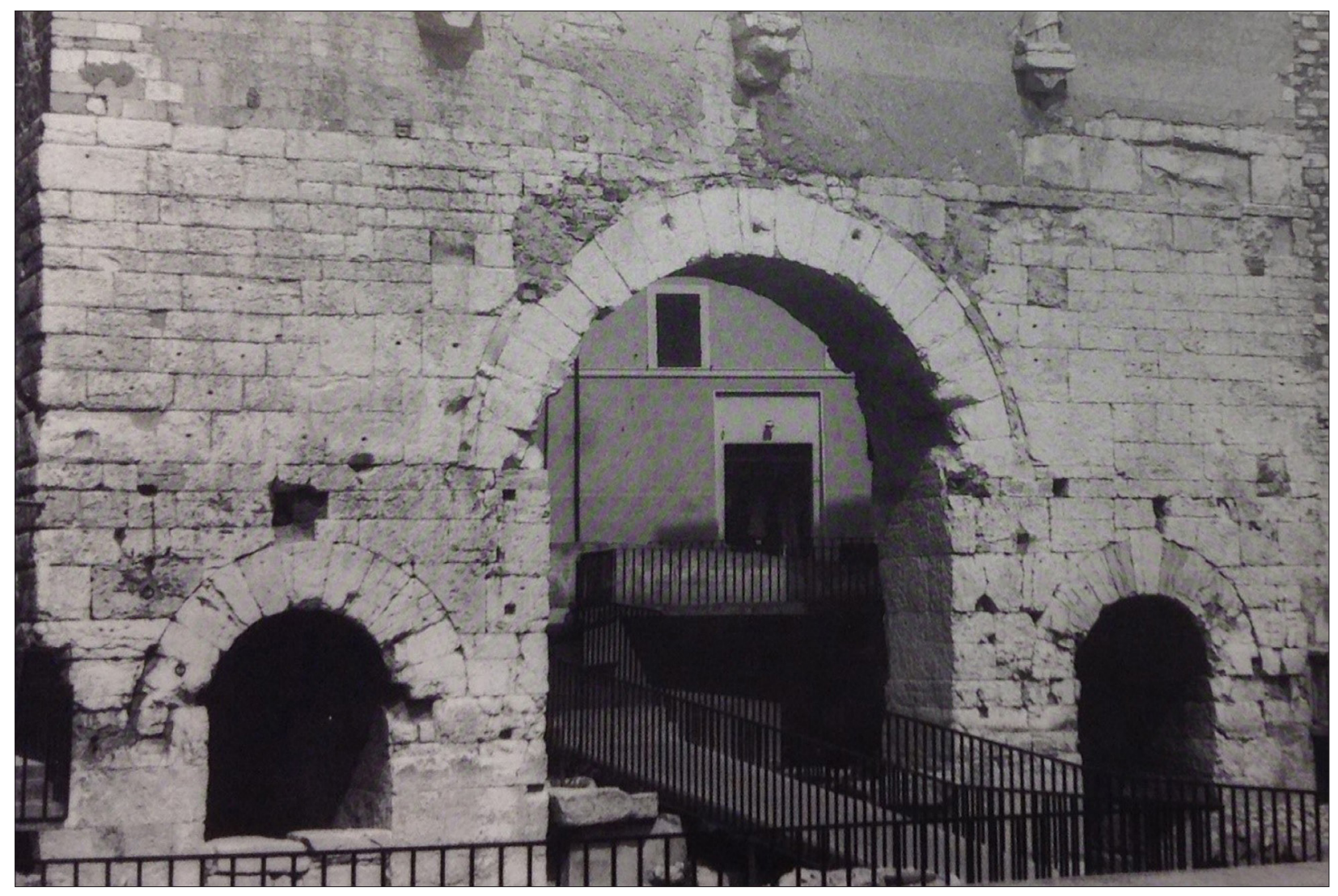

Fig. 3. Spello (Hispellum). Porta Consolare. Prospetto esterno (da Baiolini, 2002: 83).

murarie, che le due città hanno in comune, è il ruolo di controllo della linea tratturale in modo da assicurarsi la continuità di proventi daziari e salvaguardare la comunità dall'eventualità, non rara, di sommosse e ribellioni armate di pastori. Saepinum si presenta come un centro di servizi civici e commerciali per una comunità extraurbana più ampia; questo assetto territoriale, fondato sul frazionamento capillare dello stanziamento umano, corrisponde perfettamente alla tipologia insediativa che si era venuta definendo precedentemente. Caso del tutto analogo è quello di Peltuinum, anch'essa attraversata dal tratturo; qui il progetto iniziale della cinta urbana si deve probabilmente ad un clima politico in fermento, ricollegabile sia ai postumi delle ribellioni schiavili sia alla verosimile persistente presenza nell'area, di seguaci di Catilina (morto nel 62 a.C.), che il senatore, ancora in vita, aveva trovato anche nella vicina Amiternum. A questo deve aggiungersi il problema dei rapporti tra pastori e agricoltori, spesso bellicosi e pericolosi. Questo particolare clima si pone come ulteriore plausibile spiegazione per il disegno accurato del perimetro murario e per il suo prolungato utilizzo.

Anche con l'avvento della pacificazione augustea, il problema della sicurezza doveva essere ancora particolarmente sentito, tanto che, per cercare di arginarlo, lo stesso Augusto decise che fossero impiantati dei presidi militari (stationes militum), che facevano capo al principe in persona, nelle aree maggiormente infestate dal brigantaggio (Laffi, 2007: 83).

In alcune aree, dunque, era ancora necessario difendersi ma, in generale, non è più quella difensiva la funzione primaria delle cinte murarie. Può affermarsi, infatti, che la cinta muraria ha adesso la sua principale ragione di esistere poiché struttura che ha il compito di definire lo spazio urbano della città e le conferisce un determinato ruolo, e spesso un prestigio, in quanto singola unità urbana. Le città assegnavano al carattere monumentale della cinta 


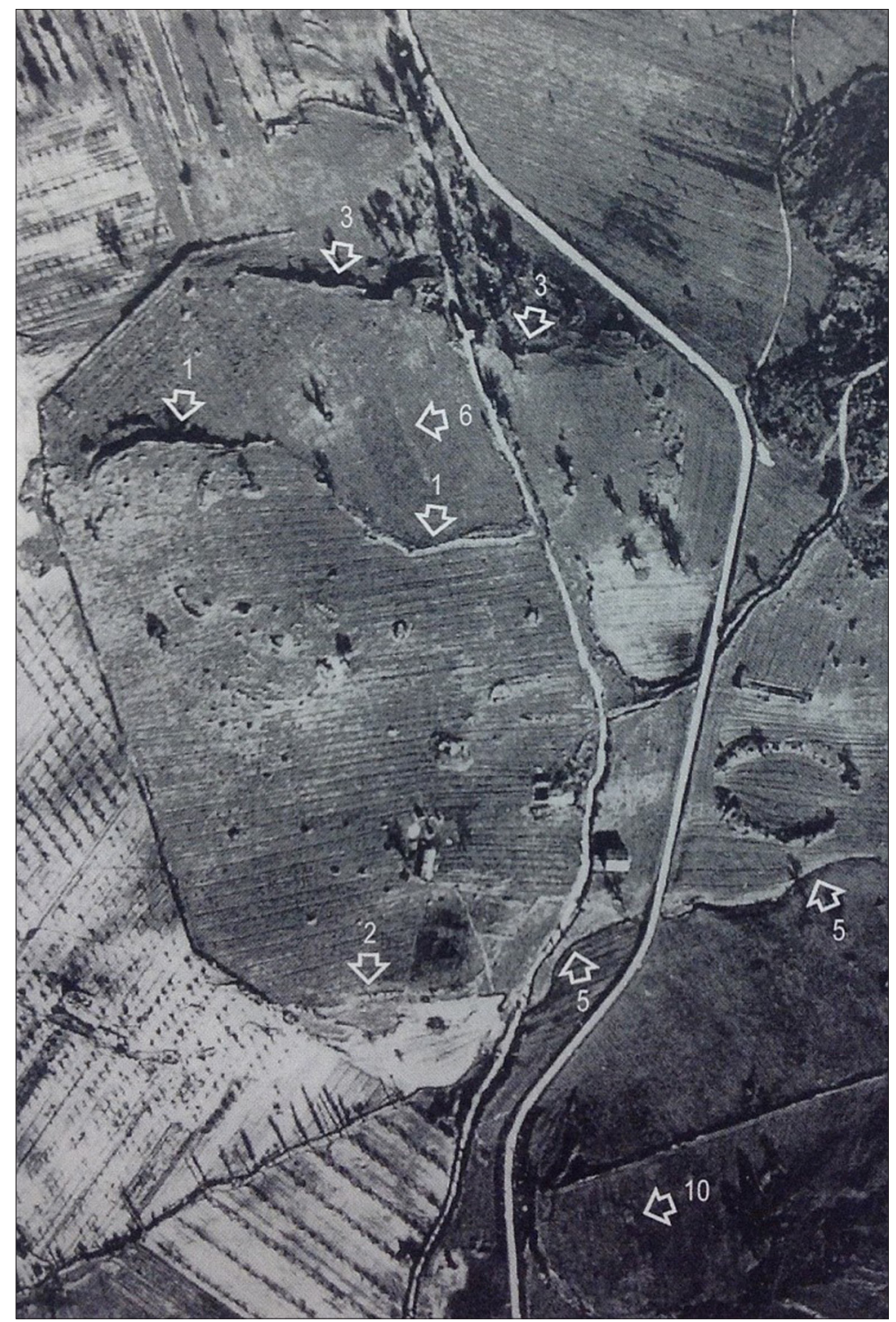

Fig. 4. Carsulae. Fotografia aerea in b/n effettuata tra il 1944 e il 1951. Le frecce indicano le tracce di muratura che potrebbero riferirsi a porzioni di cinta muraria e che oggi non sono più ben leggibili a causa del rimboschimento e dei grandi sconvolgimenti agrari che hanno interessato la campagna italiana sin dagli anni '50 (da Ciuchini, 2004:123).

urbana, all'altezza e al numero delle torri e all'eleganza architettonica delle porte, l'espressione della propria forza e della propria potenza; potenza che poteva essere di natura politica o commerciale. Ciò divenne così connaturato nella mentalità del mondo romano, che spesso le fonti usano il termine moenia, che indica precisamente la cinta muraria, come sinonimo del termine città.

Una minore attenzione alla funzione difensiva si riscontra in quelle città dove si configura un aumento del numero e delle dimensioni dei fornici delle porte o anche dove non vi sia traccia di sistemi di chiusura, come nel caso dell'arco di Augusto di Ariminum (Foschi \& Pasini, 1998; Mansuelli, 1960; Ortalli, 1995). Tale struttura (Fig.
2) non si prestava di certo, per l'ampiezza del fornice, ad esigenze difensive e, soprattutto, non prevedeva più alcun sistema di chiusura; il fornice, infatti, non presenta traccia di battenti o di solchi per cataractae e, dunque, doveva rimanere sempre aperto. La sostituzione di una porta con un arco onorario ha causato anche la mancanza di comunicazione della nuova struttura con le due torri laterali di difesa, interrompendo il camminamento di ronda della cinta urbana. Il significato simbolico e monumentale di questa struttura come terminale della via Flaminia va oltre il valore urbanistico di confine della città.

Nel caso di Novum Comum, invece, soltanto una torre losservabile nei sotterranei dell'Istituto Mag- 


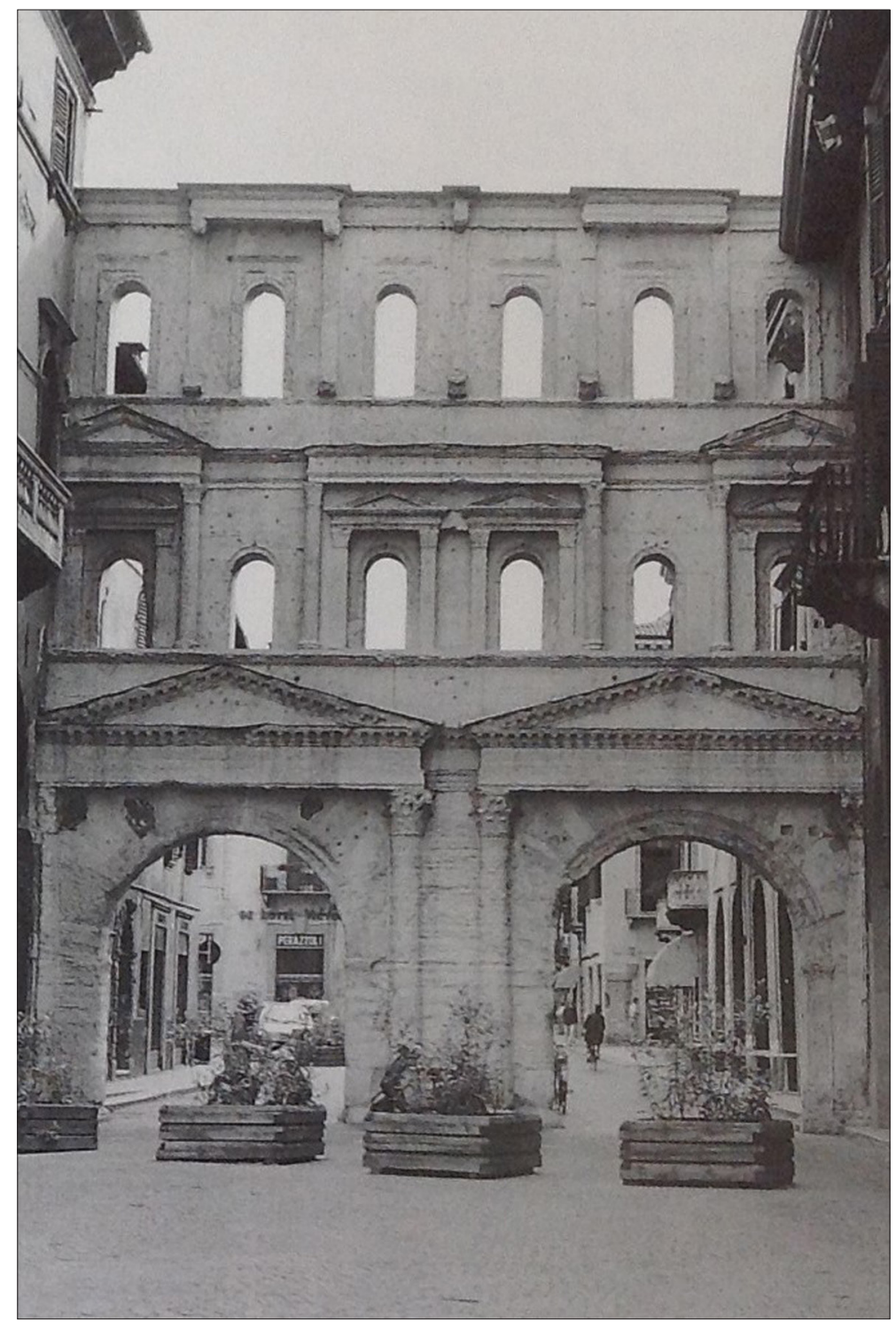

Fig. 5. Verona. Il paramento marmoreo di età claudia di porta Borsari all'ingresso occidentale della città (da Conforti Calcagni, 1999: 31).

istrale) su otto finora rinvenute, appare coeva alle mura e organicamente inserita in quelle (Maggi, 1993a; 1993b). La costruzione delle torri, dunque, è stata fatta in momenti successivi, pericolosi per la città, in modo precipitoso, come si evince anche dall'uso, in esse, di materiali architettonici e scultorei eterogenei, provenienti da edifici più antichi. Questo attesta la necessità di rendere militarmente sicura una cinta che non lo era prima, poiché chiamata a svolgere, fino a quel momento, una funzione unicamente rappresentativo-simbolica. In caso di pericolo, infatti, si deve costruire con tutto ciò che si ha a disposizione. Quando non si ha fretta, si può scegliere cosa utilizzare, come nel caso di Ariminum, dove, per la costruzione dell'arco e del ponte lungo la via Emilia si importò pietra dall'Istria.

Nelle porte urbiche si continua, comunque, a mantenere il carattere intrinseco di opere militari anche grazie all'inserimento, nel circuito murario, di torri aggettanti; ma il corpo stesso della porta diviene, a volte, un vero e proprio monumento onorario o commemorativo di particolari eventi. Il primo esempio di questa categoria è il già citato arco augusteo di Ariminum, la sua funzione di porta urbica è ripetuta anche dalla più tarda porta di Ticinum, del 7-8 d.C., di analoga destinazione onoraria, ma in senso dinastico, di cui, tuttavia, non ci resta che il testo dell'iscrizione, conservatoci dall'Anonimo di Einsiedeln (CIL V 6416).

Una connotazione analoga potrebbe essere 
accettata anche per l'arco a un solo fornice, detto di Riccardo, a Tergeste. Considerando la sua posizione, che lo doveva porre in diretto contatto con l'area del foro alla sommità del colle di S. Giusto (Rosada, 2009: 479), potrebbe non essere, infatti, da escludere una sua destinazione onoraria (Rosada, 1990: 395).

Altri esempi significativi di porte urbiche - archi onorari sono: la porta Boiano di Saepinum, l'arco di Augusto a Fanum Fortunae, la porta Consolare di Hispellum (Fig. 3), le porte Praetoriae di Augusta Prætoria Salassorum e di Augusta Taurinorum. Una gamma monumentale che manifesta, nelle grandi dimensioni del fornice unico o nel moltiplicarsi del numero di passaggi, un distacco, che diventa progressivamente sempre più evidente, da quella che era la loro funzione originaria.

Tra le porte con passaggi multipli, vediamo che la continuità tipologica della porta Leoni di Verona colloca alla fase triumvirale il primo impianto a doppio fornice, e ribadisce, nel momento del completo rinnovamento augusteo, l'avvenuta assimilazione delle porte duplici tra le costanti monumentali caratterizzanti l'acquisizione della dignitas urbana (Cavalieri Manasse, 1993; Conforti Calcagni, 1999; Forlati Tamaro, 1965; Polacco, 1974). Tra i non numerosi esempi di porte gemine si impone il concetto dell'evidenziazione dell'asse urbano principale; questo avviene sia nel caso di Asculum (Luni, 2000a), che viene attraversata dalla Salaria, sia in quello di Peltuinum, attraversata dalla Claudia Nova, viabilità definita nel 43 d.C. ma assestata su una viabilità zonale che possedeva grande rilievo già nella immediata fase pre-imperiale. Il significato urbanistico, dunque, riconosce nell'acquisizione delle porte monumentali anche un criterio di gerarchizzazione viaria; intesa nel senso di fornire importanza agli assi portanti dell'impianto urbano fin dalle fasi iniziali dell'attuazione del progetto edilizio della città stessa.

In età augustea a Ravenna le mura persero completamente la loro funzione militare, come mostra il loro parziale utilizzo da parte di edifici privati (Fabbri, 2004). Un ulteriore indizio di questa situazione è dato dal rifacimento da parte dell'imperatore Claudio, nel 43 d.C., della porta urbica, che controllava l'accesso in città dal porto, e che quindi muniva una delle entrate più importanti. Anche in questo caso, come si è visto per Ariminum, la struttura di una porta venne sostituita con quella di un arco onorario. L'intervento di Claudio, documentato solo per via antiquaria da una serie di disegni, venne distrutto nel corso del XVI secolo. Recenti scavi, tuttavia, hanno permesso di mettere in luce come non si trattasse di un rifacimento vero e proprio, ma semplicemente dell'applicazione di una struttura di rivestimento in marmo, che ricopriva e modificava l'aspetto della precedente struttura in laterizio. La visione dell'arco, dunque, veniva a contrastare con quella delle due torri vicine, che mantenevano l'aspetto originario del laterizio.

I casi di perimetri quadrangolari come quelli di Augusta Taurinorum e Augusta Prætoria Salassorum, ancora oggi, spesso, sono presentati come rispondenti a reali esigenze militari e derivanti direttamente da impianti castrensi. In questi casi si può, piuttosto, pensare ad un concepimento rispondente ad esigenze urbanistiche di regolarizzazione, non tralasciando, inoltre, l'aspetto, non meno rilevante, della semplicità e rapidità love la morfologia del terreno, ovviamente, lo consentissel di progettazione ed esecuzione. Tali insediamenti regolari instauravano, infatti, tra spazio libero rurale e spazio strutturato urbano un rapporto di regolarizzazione, interdipendenza e complementarietà, allo stesso tempo reale e simbolico, attraverso l'uso sistematico e cadenzato di forme geometriche regolari. Anche le torri potevano avere il compito di svolgere una funzione urbanistica; in città dall'impianto regolare, infatti, come, ad esempio, Augusta Taurinorum e Tridentum (Bassi, 2005), un osservatore, che si fosse posizionato in un qualunque incrocio della città, poteva intercettare una torre, o una porta, al fondo di ogni via; queste, infatti dovevano svolgere un ruolo di funzionale punto di riferimento urbanistico, divenendo, insieme alle porte, ciascuna l'indicatore di un diverso asse dell'impianto urbano, ponendosi, appunto, in asse con la viabilità interna.

Se in centri come Aosta e Torino si può rilevare una certa regolarità geometrica del perimetro 
urbano, a cui corrisponde anche una regolarità dell'impianto interno, condizionamenti più precisi, pur conservando un assetto ordinato e ortogonale degli isolati, si riscontrano nelle cinte di altri centri importanti come, ad esempio loltre la già citata Verona), Brixia, il cui circuito (di cui sopravvivono $250 \mathrm{~m}$ circa, ossia meno di un decimo del totale) fu realizzato in occasione del cambiamento dello stato giuridico (Colonia Civica Augusta nel 27 a.C.), possedeva una pianta pentagonale irregolare che si adattava all'orografia e racchiudeva una superficie di 50 ettari, più di metà dei quali erano coperti dall'area edificata, che occupava in parte anche il declivio del colle Cidneo (Breda, 1993; Stella, 1998).

Un adattamento soprattutto alle caratteristiche orografiche dei siti si riscontra anche a Tergeste e Pola. Entrambe le città si sviluppano su una collina lambita dal mare. Della specifica morfologia tengono conto l'abitato e lo stesso circuito di mura urbiche, disposto a seguire la conformazione del rilievo (le due cinte sono attribuibili al decennio tra 40 e 30 a.C.). A Tergeste (Maselli Scotti, 1990; 2006) sembra riscontrabile l'intenzione di sfruttare in senso scenografico lo stesso assetto morfologico/ urbano, che trovava riferimento nel colle di S. Giusto, nella sua sistemazione come area capitolina (circa alla metà del I sec. d.C.) e nella costruzione del teatro, durante la prima età traianea (Rosada, 2009). Pola ha una pianta racchiusa in cui gli isolati della città bassa si disponevano ordinati e regolari mentre quelli collinari si adattavano necessariamente al terreno.

In alcuni casi, si può anche pensare a una funzione, svolta dalle mura, come elemento urbanistico di zonizzazione. Ad esempio, ad Alba Pompeia, in base ai rinvenimenti archeologici è stata avanzata l'ipotesi (Filippi, 1997) che il settore meridionale della città vedesse una maggiore presenza di attività commerciali e artigianali, in contrasto con la zona settentrionale dove, finora, sono concentrate tutte le residenze di maggior pregio. La zona meridionale appare direttamente connessa, attraverso l'asse NS, con l'agro meridionale e sud-occidentale, senza il problema del superamento dei corsi d'acqua. Il settore settentrionale della città appare, invece, più isolato, bloccato a sud dall'area pubblica e sugli altri lati dalle mura (Filippi, 1997: 66), che svolgerebbero, dunque, una funzione urbanistica di zonizzazione. Ad Augusta Taurinorum, si presenta una situazione analoga nel settore nord-ovest dell'impianto romano, in connessione con il lato della città prospettante il fiume (la Dora Riparia), dove sono state individuate domus di un certo pregio. Si potrebbe avanzare, dunque, l'ipotesi di una gerarchia nella destinazione dei quartieri residenziali e di una scala nei valori catastali delle aree urbane, dove fossero privilegiati alcuni quartieri residenziali da tenere più appartati, lontani dal traffico, dal rumore, dal sudiciume e dalla delinquenza urbana, che anche in antico, caratterizzavano le aree urbane più popolari e affollate.

Si è già accennato al concetto di rapporto tra spazio libero rurale e spazio strutturato urbano, concetto che è necessario approfondire ulteriormente. Le mura, da sempre apparato difensivo e utilitario per eccellenza, continuano ad essere rappresentanti di una barriera fisica, dotata di una propria specifica imponenza e carica ideologica, che si pone tra la città e la campagna. In questo contesto, le porte urbiche, si pongono come collegamento tra città e campagna, distinguendosi, in questo modo, dalla cinta muraria, che può anche essere costruita in un secondo momento 0 , addirittura, non essere mai realizzata; anche se la realtà archeologica odierna non ci fornisce esempi certi di insediamenti che in antico fossero privi di mura ma provvisti di porte monumentali. A Libar$n a$, in effetti, il perimetro della città era segnato da porte ma, al momento, non è possibile affermare la presenza o meno delle mura (Preacco, 2008; Zanda, 2004). Probabilmente Lucus Feroniae e Veleia non ebbero mai una cinta muraria (Sommella, 1988: 161); mentre per quanto riguarda la città di Carsulae (Fig. 4), questa è stata a lungo ritenuta esempio di città "aperta" delimitata da un arco-porta monumentale ma le fotografie aeree, che già suggerivano la presenza delle mura, sembra abbiano trovato oggi conferma in un'attività di ricognizione svolta nel decennio scorso (Ciuchini, 2004). Può ricordarsi, inoltre, il caso di Colonia Augusta Bagiennorum, che non ebbe mai una cinta 
in muratura, bensì unicamente porte in corrispondenza delle strade di accesso, torri d'angolo e un sistema difensivo costituito da palificate lignee e da un vallum-fossato, che, a quanto sembra, cadde in disuso già in epoca antica e pare avesse anch'esso uno scopo più "simbolico-rituale" che protettivo (Preacco, 2014). Abbiamo, però, casi certi di cortine costruite dopo la costruzione delle porte, come, ad esempio, ad Augusta Taurinorum, dove le cortine si sono appoggiate alle torri e, dunque, queste ultime le di conseguenza anche le portel sono state costruite prima della cinta fortificata.

La porta, dunque, diventa il punto di collegamento tra spazio rurale e spazio urbano e lo fa entrando in simbiosi con l'arco; un monumento civile che Augusto recupera e pone all'interno della sfera religiosa, svuotandolo del suo significato trionfale e facendolo divenire un monumento ufficiale e rappresentativo, diventando, in questo modo, parte integrante del programma augusteo di rinnovamento culturale (Mansuelli, 1979). Tra gli esempi, ci sono casi di ristrutturazione, come quello della città di Fanum Fortunae (Luni, 2000b), che mostrano chiari i segni di propaganda e di considerevole munificenza da parte dello stesso Augusto, che si esplica anche nella edificazione della cinta difensiva volutamente enfatizzata nella monumentalizzazione degli ingressi. A Dertona (Bargnesi, 2006; Finocchi, 2002; Maggi, 2006), avviene precocemente lo scadimento della funzione difensiva della cinta muraria, che era stata realizzata verso la fine del II o inizi del I sec. a.C., ma questa viene ripristinata già a cavaliere tra il I sec. a.C. e il I d.C. A Firmum Picenum (Pasquinucci, 1987), il perimetro murario originario, risalente all'impianto della colonia latina, deve aver subito inevitabili restauri ed eventuali rimaneggiamenti tra la fine dell'età repubblicana e la prima età imperiale, in occasione delle imponenti opere di ristrutturazione della città determinate dalla deduzione di veterani della Quarta Legio. Anche l'intera cinta muraria di Trebiae (Marchi, 2002; Sensi, 1974) è oggi posta in un ambito cronologico oscillante tra l'inizio del I sec. a.C. e l'inizio del I sec. d.C.

Le mura del periodo augusteo presentava- no negli alzati prospetti lapidei o in mattoni. Lo specifico impiego di mattoni o pietre, tra loro diversi, per struttura, composizione e effetti cromatici, faceva assumere all'intero circuito murario una differente conformazione strutturale e coloristica, conferendogli connotati architettonici e linguaggi figurativi diversi, sia nella forma sia nell'articolazione. Una rilevante differenziazione cromatica e strutturale si trova, ad esempio, nella Porta Venere di Hispellum, nella Porta Palatina di Augusta Taurinorum, nella scomparsa Porta Aurea di Ravenna, nell'arco di Augusto a Fanum Fortunae e nella Porta Veronensis di Tridentum (Baggio Bernardoni, 1998-1999), che con il suo cromatismo rosso e bianco doveva risaltare notevolmente sul differente e scadente materiale utilizzato per le torri.

In questi anni emerge l'elemento spaziale della prospettiva e l'esempio più significativo proviene da Verona. Proprio in questa città, infatti, i rapporti tra mura e soprattutto porte, tessuto urbano interno e viabilità esterna si possono cogliere in termini evidentissimi. Qui, all'interno di un'ansa dell'Adige e sull'asse della Postumia si sviluppò, in epoca tardo repubblicana e nel I sec. a.C., il piano urbanistico della città, protetta dalle mura sui lati sud-orientale e sud-occidentale. Su quest'ultimo si apriva la Porta lovia, oggi Borsari (Fig. 5), a cavaliere della Postumia; sull'altro lato protetto dalla cinta si trovava la Porta Leoni che individuava un secondo asse urbano in aggiunta alla strada consolare: entrambi gli assi convergevano nello spazio destinato alla piazza forense che diventava un fulcro congiuntivo, segnalato a sua volta da un ulteriore arco (di Giove Ammone) poco oltre Porta Borsari. Successivamente, con la costruzione dell'arco onorario della gens Gavia, in epoca tardo augustea-tiberiana, sembra completarsi l'immagine di Verona nel suo rapporto tra zona urbana interna e definita e territorio esterno. L'arco infatti si pone sulla Postumia a circa $550 \mathrm{~m}$ di distanza dalla Porta Borsari e si doveva proporre in sintonia con la porta urbica come un prolungamento dello stesso ingresso aperto nelle mura, una sorta di propileo che annunciava dalla campagna la città in chiave monumentale. All'altro capo del tratto 
urbano della Postumia, dove questa attraversava l'Adige, si trovava un'altra architettura archivoltata costituita dal cosiddetto ponte Postumio loggi non più conservatol, che veniva così a corrispondere in qualche modo all'Arco dei Gavi (Rosada, 1990: 383 ss). Un sistema così strutturato trova riscontri anche in altri centri che propongono soluzioni in parte assimilabili. Tra questi, vi è il caso di Aquinum, con la porta orientale e il ponte su una strozzatura dei tre laghetti (o probabilmente paludi) formati un tempo dal torrente Le Sogne (prosciugato nel Cinquecento). La linea delle mura risulta coerente con la rete stradale urbana e secondo questa orientata nei lati settentrionale, occidentale e meridionale. Per quanto riguarda la chiusura del lato orientale, che era delimitato dai laghetti antichi di cui ancora si vedono gli alvei (Murro, 2010: 23), questa è stata a lungo dibattuta ma oggi è concordemente accettata. Anche il lato orientale, dunque, era protetto da una fortificazione che doveva seguire l'andamento del ciglio del Vallone e, accettando questa ipotesi, tutto il settore ad est è da inserire all'interno dell'area urbana e diviene parte integrante della città antica, una sorta di acropoli che si affacciava sui laghi e dominava da una posizione favorevole l'intera estensione cittadina (Ceraudo, 2004: 17).

A Hispellum il gusto per la prospettiva si esprime nella facciata palaziale (come la Porta Borsari di Veronal che marca il diaframma delle mura, quasi a suggerire le architetture stesse della città. A Hispellum si aggiunge anche l'arrivo in salita della strada, un diverticolo della via Flaminia, che proietta alta la prospettiva della Porta Venere tra due torri (Manconi, 1996).

A Fanum Fortunae Augusto costruisce una porta-arco monumentale a cavaliere dell'ingresso in città della bretella della via Flaminia. La strada trova in questa struttura l'enfatizzazione del suo passaggio e del rapporto diretto con la forma urbis; un rapporto che a Fanum Fortunae acquista un valore rilevante poiché la stretta coerenza tra impianto regolare della città e agro centuriato fa rientrare la programmazione urbana e territoriale nello stesso progetto.

Nel contesto delle mura, gli ingressi alla città costituivano scenari prospettici obbligati e assumevano, nel paesaggio suburbano, un primario rilievo come elementi polarizzatori di visuali prospettiche. Le porte, definivano in senso architettonico e simbolico, il punto d'incontro tra città e campagna, pianificazione urbana e pianificazione rurale. In questo contesto, la valorizzazione dell'area extraurbana come bene d'uso sposta il campo di attenzione e di rappresentatività anche all'esterno, al territorio e a ciò che da questa prospettiva si poteva scorgere della città.

Non è più necessario, dunque, che l'elemento mura debba chiudere l'abitato in una rigida alternativa interno/esterno, chiuso/aperto. Al contrario, si può rilevare un rapporto costante e dialettico tra la realtà insediativa e quanto le sta intorno e il collegamento, oltre che attraverso le porte le di conseguenza le strade), può avvenire anche attraverso altre strutture, sia antropiche, come ad esempio i ponti, sia naturali, come ad esempio i corsi d'acqua.

Il moltiplicarsi dei punti di collegamento tra interno ed esterno evita la scelta di un solo prospetto prevalente della città, e rivaluta l'immagine urbana nella sua tridimensionalità e nella pluralità delle sue saldature con il territorio. Un tale procedimento è attuato da Augusto ad Ariminum, costruendo ad oriente l'arco-porta urbica sulla via Flaminia, e a occidente il ponte lungo la via Emilia. Due monumenti tipologicamente diversi, ma di cui non era difficile percepire l'unitarietà della concezione lanche per lo stesso contrasto che li caratterizzava ottenuto da un diverso materiale e colore rispetto alla cortina laterizia delle mural e la complementarietà nella definizione visiva del centro urbano e dei suoi nessi con il territorio. Archi esterni e ponti, nella loro funzione di connessione con il suburbio, appaiono appoggiati all'immagine urbana, che si profila come sfondo per chi si avvicina alla città.

Ad Augusta Prætoria Salassorum l'arco del 25 a.C. sorge a circa $400 \mathrm{~m}$ dalla Porta Praetoria, sulla via per Eporedia; l'arco è situato sulla riva del torrente Buthier, dove si conservano i resti (Pont de Pierrel del ponte coevo. A Verona, il più tardo arco dei Gavi (Tosi, 1983), si situa extra moenia lungo la 
via Postumia, mezzo chilometro fuori Porta Borsari, sul ciglio del terrazzo alluvionale su cui sorgeva la città, marcato anche dall'avvallamento di un antico ramo dell'Adige.

\section{Bibliografia}

Baggio Bernardoni E, 1998-1999: La porta Veronensis a Trento, in Archeologia veneta TEXNH. Studi di urbanistica greca e romana in onore di Giovanna Tosi. 21-22: 95-109. Società archeologica veneta ONLUS, Padova.

Baiolini L, 2002: La forma urbana dell'antica Spello, in Città dell'Umbria. Atlante tematico di Topografia Antica XI supplemento-Città romane 3: 61-119. L'Erma di Bretschneider, Roma.

Bargnesi R, 2006: Tortona in età romana. Il territorio. L'assetto del territorio di Dertona, in Dertona Historia Patriae. Storia di Tortona dalla preistoria a oggi. L'età romana, II sec. a.C.-V sec. d.C. vol. II: 39-57. Società Storica Pro lulia Dertona, Tortona.

Bassi C, 2005: Trento romana. Un aggiornamento alla luce delle più recenti acquisizioni, in Ciurletti $\mathrm{G} \&$ Pisu N (ed.), I territori della via Claudia Augusta: incontri di archeologia: 271-88. TEMI, Trento.

Breda A, 1993: Le mura di Brixia, in Mura delle città romane in Lombardia. Atti del convegno: 83- 97. Società archeologica comense, Como.

Casazza G \& A Pansini (in corso di stampa): Un nuovo settore delle fortificazioni, in Quaderni di Archeologia d'Abruzzo, All'insegna del Giglio, Sesto fiorentino (FI).

Cavalieri Manasse G, 1993: Le mura di Verona, in Mura delle città romane in Lombardia. Atti del convegno: 179-215. Società archeologica comense, Como.

Ceraudo G, 2004: Aquinum: la città romana, in Ceraudo $\mathrm{G}$ (ed.), Ager Aquinas. Aerotopografia archeologica lungo la valle dell'antico Liris: 13-23. Caramanica Editore, Marina di Minturno (LT).

Ciuchini P, 2004: Carsulae: alcune notazioni in merito ai resti della cinta di fortificazione e alle tracce da foto aerea ad essa riferibili, in Ceraudo G \& F Piccarreta, Archeologia aerea. Studi di aerotopografia archeologica: 119-26. Libreria dello Stato - Istituto poligrafico e zecca dello Stato, Roma.

Conforti Calcagni A, 1999: Le mura di Verona. La città e le sue difese dalla fondazione romana all'unità d'Italia, Cierre, Caselle di Sommacampagna.

Fabbri P, (ed.) 2004: Le mura nella storia urbana di Ravenna, Società di studi ravennati, Ravenna.

Filippi F, (ed.) 1997: Alba Pompeia: archeologia della città dalla fondazione alla tarda antichità, Famija Albèisa, Alba (CN).

Finocchi S, 2002: Iulia Dertona Colonia, Edizioni Oltrepò, Voghera.

Forlati Tamaro B, 1965: Verona. Il restauro della Porta detta dei Leoni, in Notizie degli scavi di antichità VIII, 19, suppl.: 12-34. Roma.

Foschi PL \& PG Pasini 1998: L’Arco di Augusto. Significati e vicende di un grande segno urbano, Musei Comunali, Rimini.

Laffi U, 2007: Colonie e municipi nello stato romano, Edizioni di storia e letteratura, Roma.

Luni M, 2000a: Monumenti di Asculum, in relazione alla Salaria, in Catani E \& Paci G (ed.), La Salaria in età antica. Atti del convegno di studi, Ascoli Piceno-Offida-Rieti 1997: 332-39. L'Erma di Bretschneider, Roma.

Luni M, 2000b: Studi su Fanum Fortunae, QuattroVenti, Urbino.

Maggi P, 1993a: Le mura romane di Como, in Mura delle città romane in Lombardia. Atti del convegno, Como 1993: 37-46. Società archeologica comense, Como.

Maggi S, 1993b: Como romana: la forma urbis. Problemi e proposte di studio, in Sena Chiesa G, Donati PA \& Sartori A led.), Novum Comum 2050. Atti del convegno celebrativo della fondazione di Como romana: 163-84. Società Archeologica Comense, Como.

Maggi S, 2006: Dertona: la città romana e la sua immagine, in Dertona Historia Patriae. Storia di Tortona dalla preistoria a oggi. L'età romana, II sec. a.C.-V sec. d.C. vol. 2: 129-47. Società Storica Pro Iulia Dertona, Tortona.

Manconi D, P Camerieri \& V Cruciani 1996: Hispellum: la sua pianificazione urbana e territoriale, in Bonamente $G$ \& Coarelli F (ed.), Assisi e gli Umbri nell'antichità. Atti del convegno Internazionale, Assisi 1991: 375- 429. Società editrice Minerva, Assisi.

Mansuelli GA, 1960: Il monumento augusteo del 27 a.C. Nuove ricerche sull'Arco di Rimini, Arti grafiche Tamari, Bologna.

Mansuelli GA, 1979: Fornix e Arcus. Note di termino- 
logia, in Studi sull'arco onorario romano: 15-17.

L'Erma di Bretschneider, Roma.

Maselli Scotti F, 1990: Tergeste, in Aquileia e l'arco adriatico. Settimana di studi aquileiesi. Antichità altoadriatiche 36: 333-45. Centro di antichità altoadriatiche (Aquileia), Arti grafiche friulane, Udine.

Maselli Scotti F, 2006: Le cinte di Tergeste romana, in Atti e memorie della Società istriana di archeologia e storia patria. volume 106, 54 della nuova serie: 519-660. Società Istriana di Archeologia e Storia Patria, Trieste.

Matteini Chiari M (ed.) 1982: Saepinum. Museo documentario dell'Altilia, Soprintendenza archeologica e per i beni ambientali, architettonici, artistici e storici del Molise, Università di Perugia, Istituto di archeologia, Campobasso.

Migliorati L, 2014: Gli scavi di Peltuinum, in Bourdine S \& V D'Ercole (ed.), I Vestini e il loro territorio dalla Preistoria al Medioevo: 249-60. École francaise de Rome, Roma.

Migliorati L \& D Canino 2014: Note di topografia vestina, in Scienze dell'Antichità 20.1, 125-37.

Murro G, 2010: Monumenti antichi di Aquino: la Porta San Lorenzo e il cosiddetto Capitolium, Museo della Città, Aquino.

Ortalli J, 1995: Nuove fonti archeologiche per Ariminum: monumenti, opere pubbliche e assetto urbanistico tra la fondazione coloniale e il principato augusteo, in Calbi A \& Susini G (ed.), Pro poplo ariminese: 469529. Fratelli Lega Editori, Faenza.

Pasquinucci M, 1987: Documentazione archeologica e impianto urbano, in Polverini L (ed.), Firmum Picenum 1: 259-95. Giardini, Pisa.

Polacco L, 1974: Lettura critica delle porte romane di Verona, in II territorio veronese in età romana. Convegno, Atti: 1-17. Accademia di agricoltura scienze e lettere di Verona, Verona.

Preacco MC, 2008: Scavi e ricerche nell'area archeologica di Libarna (1960-1989), in Rossi G, M Venturi Gambari \& Zanda E (ed.), La riscoperta di Libarna. Dall'antiquaria alla ricerca archeologica, Atti del convegno (Genova 2004); 125-33. Ferrari, Genova.

Preacco MC, 2014: Augusta Bagiennorum. Storia e archeologia di una città augustea, Celid, Torino.

Rosada G, 1990: Mura, porte e archi nella decima regio: significati e correlazioni areali, in La città nell'Italia settentrionale in età romana. Morfologie, strutture e funzionamento dei centri urbani delle regiones $X$ e XI, Atti del convegno, Trieste 1987: 365-409. CEFR 130, Trieste-Roma.

Rosada G, 2009: Continuità e trasformazione dei fora. I casi post-classici di Verona, Vicenza e Trieste, in

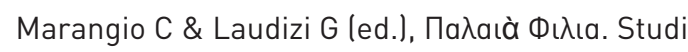
di topografia antica in onore di Giovanni Uggeri: 46586. Mario Congedo editore, Galatina.

Sensi L, 1974: Trebiae, in Castagnoli F, Ricognizione archeologica e documentazione cartografica: 183-90. De Luca editore, Roma.

Sommella P, 1988: Italia antica. L'urbanistica romana, Jouvence, Roma.

Stella C, 1998: I dati archeologici di Brixia, in Brescia. L'età romana: 26-27. Electa, Milano.

Tosi G, 1983: L'arco dei Gavi, L'Erma di Bretschneider, Roma.

Zanda E, 2004: Libarna, Allemandi, Torino. 